\title{
Reseña de libro: \\ El populismo en escena ¿Por qué emerge en unos países y en otros no? de César Ulloa
}

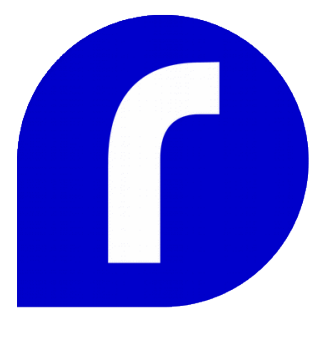

Ulloa, César. 2017. El populismo en escena ¿Por qué emerge en unos países y en otros no? $1^{\mathrm{a}}$. Quito: FLACSO-Ecuador.

\begin{abstract}
José Andrés Díaz González
Doctorando en Gobierno y Políticas Públicas. Mágister en Historia y Licenciado en Ciencias Políticas. Investigador del Instituto de Estudios Sociales en Población (IDESPO) de la Universidad Nacional de Costa Rica (UNA) y docente de la Escuela de Ciencias Políticas de la Universidad de Costa Rica (UCR).

Correo: jose.diaz.gonzalez@una.ac.cr y joseandres.diaz@ucr.ac.cr
\end{abstract}

\section{Resumen}

El populismo en América Latina no es un fenómeno nuevo, y tampoco es exclusivo de estas latitudes. Sin embargo, la región parece funcionar como un "laboratorio" político para estudiar el tema del surgimiento, consolidación y caída de gobiernos populistas. Dado esto, es posible realizar un análisis político comparado que permita identificar las características que facilitan que el populismo surja en algunos países latinoamericanos, mientras que en otros parecen estar exentos de este fenómeno. Este es el punto de partida de César Ulloa en su libro en su libro El populismo en escena ¿Por qué emerge en unos países y en otros no?, en el cual realiza un análisis comparado de dos países con una fuerte tradición populista en su escenario político, Venezuela y Ecuador, y los contrasta con Uruguay, un país donde no ha surgido el populismo hasta el momento.

En las siguientes páginas presento una revisión de los principales argumentos y hallazgos de Ulloa (2017) respecto al surgimiento de gobiernos populistas; pero también aprovecho la oportunidad para realizar una reflexión sobre la coyuntura política costarricense, a partir de los elementos identificados por Ulloa que facilitan el surgimiento de populismo en los escenarios políticos, para tratar de visualizar qué tan proclive se encuentra Costa Rica de tener un gobierno de corte populista. 


\section{Un acercamiento al estudio del Populismo}

El interés de Ulloa es determinar cuáles son las características políticas, sociales y económicas que facilitan o dificultan el surgimiento de gobiernos, movimientos o políticos populistas. Así, inicia su obra definiendo populismo como:

[...] una estrategia que no enfatiza lo ideológico, se caracteriza por un discurso popular-confrontacional (contra las oligarquías, las instituciones, crea permanentemente enemigos, goza de capacidad de movilización y se dirige a un segmento policlacista, pese a que enfatiza su interés en segmentos de menores recursos económicos (Ulloa 2017).

Al reconocer que el populismo es una estrategia, y no una ideología, se parte que este puede ser utilizado tanto por políticos de derecha como de izquierda. Asimismo, al caracterizarlo como estrategia, Ulloa plantea que este debe ser visualizado como un "método" o conjunto de acciones que un político (o grupo político) utiliza para obtener el apoyo popular requerido para hacerse del control del poder político de un país y, por lo tanto, no debe visualizarse como una pretensión de establecer una alternativa diferente al orden político imperante en una sociedad.

Dado lo anterior, Ulloa dedica el primer capítulo del libro a revisar cuál es la relación entre el populismo y la democracia, y cómo el primero afecta o incide en las instituciones democráticas de un país. Tras una revisión de literatura académica sobre este tema, identifica tres perspectivas a partir de las cuales se ha analizado la relación populismo-democracia: a) como un fenómeno que democratiza; b) como un fenómeno que debilita la democracia y obstaculiza el desempeño de las instituciones; y c) como un espejo de la democracia, es decir, un fenómeno que ocurre cuando la democracia no se encuentra funcionado de manera correcta o es cuestionada por la sociedad.

\section{Democracia y Populismo}

En el capítulo dos, el autor expone las principales características y desarrollo histórico de la democracia en Venezuela, Ecuador y Uruguay; aproximadamente desde mediados del siglo $X X$ y hasta finales de la primera década del siglo XXI. Aquí es necesario anotar que Ulloa utiliza el llamado método histórico de la Ciencia Política, esto con el propósito de estudiar y comprender el populismo no como un fenómeno aislado o atemporal, sino como parte y producto de procesos políticos que se gestan durante décadas en distintas so- 
ciedades. El mismo razonamiento se aplica a su análisis de la democracia venezolana, ecuatoriana y uruguaya; observa a estas como producto de una trayectoria histórica en la cual intervienen una amplia gama de actores y acontecimientos, al tiempo que deja claro que no puede observarse el desarrollo y consolidación de la democracia como un proceso lineal y teleológico, sino que tiene sus retrocesos y contradicciones. Aquí el autor empieza a identificar algunas diferencias en el desarrollo político-democrático de estos países, que parecen explicar la aparición (o no) del populismo. La primera diferencia es la consolidación de los partidos políticos; en el caso de Uruguay, tanto antes como después del régimen dictatorial existente entre 1973 y 1985, este cuenta con partidos políticos consolidados y estables, los cuales cumplen de manera adecuada con su labor de ser intermediarios y representantes de los intereses de la ciudadanía ante las instancias de gobierno. En el caso de Venezuela y Ecuador, estos no establecieron un sistema de partidos políticos fuertes y estables.

La segunda diferencia es la relación entre tecnócratas y políticos en dichos países. En el caso de Venezuela, y en menor medida Ecuador, se consolidó la designación de "técnicos" sobre "políticos" en los puestos de dirección del Estado, con lo cual tomó fuerza la implementación de políticas que disminuían el accionar de los Gobiernos en temas sociales, al mismo tiempo que se disminuía el control y liberalizaba la economía. En palabras de Ulloa:

[...] se presentó una ruptura en el monopolio de la representación política, pues los tecnócratas entraron a organizar y administrar la cosa pública, quienes carecían de toda vinculación con la sociedad, de legitimidad dentro de los partidos, pero se autoacreditaron por su formación académica y apoyo del Ejecutivo. La técnica se impuso a la política (Ulloa 2017, 90).

La tercera diferencia tiene que ver con la Cultura Política y la conflictividad social en los países analizados. En el caso de Uruguay, el respaldo de la población hacia la democracia y sus instituciones es fuerte y antiguo. En lo que respecta a Ecuador, este país nunca ha podido consolidar una fuerte afección de su población hacia la democracia. En Venezuela, si bien ha sido considerada una de las democracias más antiguas y consolidadas de América Latina, desde finales de la década de 1970 su población apoya cada vez con menos fuerza el régimen democrático. A esta situación se le debe sumar que desde la década de 1990 en Venezuela y Ecuador ha habido un importante aumento de la conflictividad social, producto de las acciones y pérdida de legitimidad de la clase política en dichos países, un fenómeno que no ha ocurrido en Uruguay.

Los capítulos 3, 4, 5 y 6 son dedicados a estudiar y comparar los políticos y gobiernos populistas en Venezuela y Ecuador. Como indique antes, Ulloa re- 
curre al llamado método histórico de la Ciencia Política para realizar su análisis. Así, el autor no aborda únicamente los casos de Hugo Chávez (Venezuela ) y Rafael Correa (Ecuador); sino que muestra cómo el populismo es un fenómeno que ha estado históricamente presente en el desarrollo políticos de estos países (y posiblemente en América Latina en general). De esta manera, analiza los casos de Abdalá Buracam, Lucio Gutierrez y Rafael Correa en Ecuador, y de Carlos Andrés Pérez y Hugo Chávez en Venezuela. Para el caso de Carlos Andrés Pérez, Ulloa hace la anotación que este no cumple con todos los elementos para ser catalogado como populista; sin embargo, su gobierno y accionar son necesarios de tomar en consideración para comprender el surgimiento y consolidación del populismo en Venezuela con Chávez.

Tras revisar las características del populismo presente en Venezuela y Ecuador, Ulloa concluye que la relación entre el populismo y la democracia es ambigua: por un lado expresa una promesa redentora para el pueblo, pero al mismo tiempo entroniza al líder y diluye todas las formas de participación horizontal que defendía durante el momento electoral. Asimismo, el populismo convierta la política en un juego de suma cero permanente; los populistas generan un discurso en donde todo aquel que se oponga o lance críticas a su acción se convierte automáticamente en su "enemigo". Esta situación provoca que se agudice la polarización política en las sociedades, dificultando que se logren tomar acuerdos políticos y minando así la capacidad de gestionar los intereses y demandas de la sociedad en un ambiente democrático (Ulloa 2017, 168-71).

En lo que respecta a las instituciones políticas, Ulloa indica que estas juegan un papel muy importante para evitar (o promover) el surgimiento del populismo. Tras revisar los casos de Venezuela, Ecuador y Uruguay, concluye que en aquellos países con instituciones débiles, cuestionadas y deslegitimadas por la sociedad es más probable el surgimiento de gobiernos populistas. Además, indica que la "extrema rigidez" de las instituciones, es decir, su incapacidad de reformarse y adaptarse a las nuevas condiciones sociales y políticas de los países, es un punto adicional que favorece el surgimiento de movimientos populistas. Precisamente el autor identifica este fenómeno para el caso de Venezuela, donde la clase política no logró alcanzar acuerdos para modificar sus instituciones para que estas dieran respuestas más adecuadas a las demandas de la sociedad. Caso contrario ocurre en Ecuador, en este país la alta inestabilidad institucional y las cambiantes reglas del juego para los actores políticos y sociales, son elementos que propician el populismo. A manera de síntesis se puede indicar que la crisis institucional, ya sea por su excesiva rigidez o por su alta inestabilidad, es un factor que propicia la llegada al poder de gobiernos populistas:

La irrupción del populismo también se explica por la inca-

pacidad de los actores políticos en recomponer el sistema político cuando las instituciones entran en crisis. En este 
sentido, se gesta un momento de inmovilidad, en el que los actores se bloquean mutuamente y eso es capitalizado por un tercero en escena que aprovecha el vaciamiento del espacio político y la falta de respuesta de los actores. Por consiguiente, el populismo también es una respuesta frente a la inexistencia de alternativas dentro del sistema (Ulloa 2017, 266).

Finalmente, Ulloa indica que la manera de hacer política del populismo se explica en la articulación e interdependencia de tres dimensiones: el discurso político, la mediatización de la política y el universo simbólico. En cuanto al discurso político, señala la importancia que tiene la apelación al "pueblo" en el discurso populista, hace un llamado a su reinvindicación al mismo tiempo que lo confronta con el "otro antagónico", con el enemigo representado por la clase política tradicional, los partidos políticos, los empresarios, los medios de comunicación, etc. El propósito del discurso populista es enardecer la confrontación y la polarización, generando así un escenario de antipolítica o ataque a todo aquello que significa el statuo quo.

En cuanto a la mediatización de la política, Ulloa establece que en Venezuela y Ecuador hay dos momentos diferenciados de este proceso. Un primer momento cuando los medios tradicionales tienen una relación cordial, e incluso puede pensarse que promueven a los líderes políticos populistas; tal sería el caso de Hugo Chávez y Rafael Correa; y un segundo momento donde estos medios pasan a ocupar el papel del "enemigo", y los gobiernos populistas promueven sus propios medios de comunicación oficiales, por los cuales buscan exaltar la imagen del Presidente. En este aspecto, el autor anota que en Venezuela y, sobre todo, en Ecuador hay una tradición personalista de la política, lo cual promueve que las fuerzas políticas se muevan alrededor de la figura de un líder, lo cual también es un factor que facilita la aparición del populismo.

Este personalismo de la política también se relaciona con el control del universo simbólico. Así, uno de los objetivos del populismo es convertir al líder en un héroe; para la construcción de esta imagen heroica se requiere que este siempre esté en batalla continua contra los opositores (enemigos). Por eso es importante para el populismo radicalizar la discusión política y convertirla en un juego de suma cero; ya que requiere la creación de antagonistas para así poder consolidar la imagen del líder-héroe.

Pero también este control del universo simbólico se realiza mediante la utilización y apropiación de íconos, fechas o figuras históricas; esto con el propósito de darles un resignificado que permita legitimarse ante la sociedad en general: 
La creación de un universo cargado de símbolos es una de las características que asemejan a Chávez y Correa. Uno de los elementos más visibles es el uso de rituales y alusiones a pasajes históricos y fechas cívicas en los cuales se trata de ensalzar a personajes que lograron la independencia y procesos revolucionarios (Ulloa 2017, 280).

Como se señaló al inicio, Ulloa establece que el populismo es una estrategia, está vació de ideología o contenido político; por lo tanto, no se presenta como una propuesta política alternativa, ni tiene un plan estructurado para mejorar las condiciones de vida del país y superar los problemas que se enfrenta; sino que en su discurso termina promoviendo nociones vagas como "refundar" o "reconstruir" el país; haciendo así alusión a una idea utópica de un pasado mejor, sin que tenga sustento en las condiciones y posibilidades reales del país. De esta manera, el discurso populista apela a la búsqueda de una "nueva institucionalidad", en la que se descartan todos los actores del pasado por considerarlos "contrarios a los intereses del pueblo", al mismo tiempo que oferta una propuesta "antiélite y propueblo", la cual finalmente cae en sus propias contradicciones al promover una relación vertical entre líder y pueblo (Ulloa 2017, 306).

\section{Algunas anotaciones para la coyuntura político-electoral costarricense}

Lo dispuesto por Ulloa en su obra, hace eco directo con la coyuntura política costarricense de cara al proceso electoral nacional de febrero del 2018; por lo que no me parece pertinente terminar estas líneas sin realizar una reflexión respecto a esta situación. En primer lugar, es necesario indicar que la actual coyuntura política costarricense no es fortuita ni circunstancial, es producto de un proceso que se puede rastrear desde finales de la década de 1990 (y tal vez antes). Desde la elección nacional de 1998 el abstencionismo en Costa Rica ha aumentado ubicándose, en promedio, en un $40 \%$. En una sociedad cuyo imaginario político tiende a visualizar que votar es sinónimo de democracia (Díaz González y Solís Salazar 2010), el aumento del abstencionismo es un reflejo de descontento y de desafección de la población hacia la política y el sistema democrático.

Asimismo, los casos de corrupción que han involucrado a altas figuras políticas, incluidos ex Presidentes de la República, son un factor adicional que promueve el alejamiento de la población hacia la política, y abona el surgimiento de figuras y discursos de la antipolítica. Hay diversos análisis empíri- 
cos sobre el comportamiento electoral y la participación política de la población costarricense que permite sostener esta idea de descontento y alejamiento de la población costarricense hacia el sistema político del país (Alfaro Redondo y Gómez Campos 2014; Díaz González 2014; Raventós Vorst et al. 2014); a lo cual es necesario sumar una reducción de la agilidad en la toma de decisiones políticas, especialmente desde la Asamblea Legislativa, debido a la existencia de un escenario político multipartidista, donde ningún actor político cuenta con la fuerza necesaria para impulsar de manera unilateral un proyecto y propuesta política; situación que se agrava por la falta de tradición y capacidad de los actores políticos de construir coaliciones políticas sólidas y duraderas (Díaz González 2011; Hernández Naranjo 2011; Borges 2014; Gómez-Campos y Villarreal 2013). Dada esta situación, no es de extrañar que la población valore de manera muy baja el desempeño del Poder Ejecutivo, del Poder Legislativo y los Partidos Políticos (CIEP 2017, 2014, 2013).

Por último, no se puede dejar de lado que la Cultura Política costarricense se caracteriza tanto por la apatía y la pasividad (Díaz González 2014), como por una fuerte disposición hacia el autoritarismo (Mora, Solís, y Soto 2014). Esto hace que la población sea receptiva a discursos políticos emanadas por figuras que se presentan así mismo como "fuertes", y con la capacidad de solucionar los problemas del país sin la necesidad del apoyo de otros actores políticos y sociales.

Llegados a este punto surge la pregunta ¿Está Costa Rica ante la posibilidad de un gobierno populista? Los elementos de la coyuntura política costarricense mencionados anteriormente son consecuentes con los factores identificados por Ulloa (2017) que facilitan el surgimiento del populismo. Por lo tanto, se puede indicar que existe la posibilidad de que un discurso populista tenga incidencia en el electorado costarricense o, al menos, en parte de este.

Antes de continuar, es necesario indicar que el populismo no es nuevo en la escena política costarricense; si bien hacen falta investigaciones para determinar si hay una tradición política populista en el país, sí es posible indicar que ha existido una serie de discursos y acciones populistas que han afectado algunas decisiones y políticas públicas que distintos gobiernos han implementado; especialmente el llamado populismo punitivo (Llobet Rodríguez 2016). Aunque la adopción de acciones y políticas que pueden ser catalogadas como "populistas" no es suficiente para determinar la existencia de una tradición de gobiernos populistas en Costa Rica, sí muestra que tanto desde la élites políticas como desde la ciudadanía, hay sectores que promueven y aceptan este tipo de acciones y, por lo tanto, pueden ser favorables a impulsar y apoyar un gobierno populista.

Por lo tanto, es posible que encontremos aspectos o propuestas en diferentes candidatos a la Presidencia de la República que compiten en las elecciones del 2018, que pueden ser catalogadas como populistas. Sin embargo, hay un candidato que perfila tener los atributos necesarios para ser identificado como populista: Juan Diego Castro. Así, Castro se posiciona como un outsider de la política nacional, esto a pesar de haber sido Ministro de Seguridad Pública y Ministro de Justicia durante la administración Figueres Olsen 
(1994-1998); en este sentido sigue una estrategia similar a la Rafael Correa, el cual se presentaba como un outsider aunque había sido Ministro de Economía.

Asimismo, el apoyo que obtiene del electorado parece que proviene principalmente por su "carisma" o imagen, que por sus propuestas políticas concretas. Tal vez la excepción es su discurso afín al populismo punitivo el cual, como ya se indicó, ha estado presente desde hace algunos años en el escenario político costarricense. Además, ha adoptado un discurso fuerte y confrontativo hacia la corrupción, lo cual ha llevado a afirmar que: "Al primer diputado que lo agarre con chorizo o se jale una torta, voy con la policía y va pal tavo" (Redacción 2017a).

También debe tomarse en consideración que Castro ha adoptado una postura antipolítica. Esto se observa tanto en los continuos ataques que realiza a la clase política, autoridades de gobierno e instituciones públicas, así como su desprecio a los partidos políticos (Cascante 2016). Incluso, Castro promueve su candidatura a la Presidencia de la República sin pertenecer a algún partido político, finalmente "negocia" con el Partido Integración Nacional (PIN) para presentar su candidatura mediante ese grupo; sin embargo, nunca ha establecido con claridad que exista una afinidad temática o ideológica entre él y dicho partido político. La falta de definición ideológica es otra característica importante del populismo, y se aprecia tanto en el discurso de Castro como en su relación con el PIN. Incluso, Castro no usa el PIN como su plataforma política (a pesar de ser su candidato presidencial), sino que se utiliza en su lugar al "Movimiento de la reconstrucción de Costa Rica", lo cual fortalece su posición antipolítica (ver https://juandiegopresidente.cr/).

Adicionalmente, el hecho de que su movimiento reciba el nombre "de la reconstrucción de Costa Rica", y que su propuesta política este basada en "Gobernar en Serio. Gobernar en Serio significa tomar las decisiones más complicadas, las que ningún otro partido político se ha atrevido [sic]" ("Un gobierno serio y enérgico" s/f), es tan bien consecuente con lo dispuesto por la política populista. Como señala Ulloa (2017), los populistas basan sus propuestas en idea de "refundar" el país, lo que implica barrer o deshacer las acciones que gobiernos anteriores han realizado por considerarlas como la causa de los problemas que enfrenta el país.

Aunque no necesariamente es una característica de los populistas, Ilama la atención que Juan Diego Castro mantiene una postura de confrontación con los medios de comunicación, al igual que Hugo Chávez, Rafael Correa y el actual Presidente de los Estados Unidos, Donald Trump. Así, Castro impide que determinados medios den cobertura a sus actividades, e incluso entra en confrontación directa con periodistas (Madrigal 2017; Redacción 2016, 2017b). Además, al igual que Donald Trump, Castro es un usuario asiduo de la redes sociales, de hecho, es mediante su página en Facebook que ha podido posicionarse ante la opinión pública como una figura "candidateable" para la Presidencia de Costa Rica. 
Regresando a la pregunta que inicia esta reflexión ¿Está Costa Rica ante la posibilidad de un gobierno populista? La respuesta apunta a que es posible que sí. En el momento de escribir estas líneas Juan Diego Castro se encuentra liderando las encuestas de intención de voto, junto a Antonio Álvarez Desanti (Partido Liberación Nacional) y Rodolfo Piza (Partido Unidad Social Cristiana), y si bien no parece que alguno de estos candidatos obtenga los votos suficientes para ser electo Presidente de la República, es muy posible que -de no haber cambios en las tendencias de voto- dos de estos candidatos se enfrenten en una segunda ronda electoral.

Por último, es necesario indicar que Juan Diego Castro no es el responsable del surgimiento del populismo en la política costarricense; al igual que todos los populistas, él simplemente está sacando provecho de las condiciones para promover su candidatura. Como indica la literatura académica, el populismo surge cuando se juntan una serie de condiciones, las cuales parecen estar presentes en la Costa Rica actual, que son producto de un proceso de años y décadas de desgaste político. Por lo tanto, si Castro no resulta electo Presidente, esto no implica que Costa Rica se libre de manera permanente de la posibilidad de un gobierno populista a futuro, este escenario solo podrá lograrse con un cambio en la gestión política del país, el cual se recupere la confianza de la población hacia la democracia y las instituciones de gobierno.

\section{Fuentes consultadas}

Alfaro Redondo, Ronald, y Steffan Gómez Campos. 2014. "Costa Rica: Elecciones en el contexto político más adverso arrojan la mayor fragmentación partidaria en 60 años". Revista de ciencia política (Santiago) $34 \quad$ (1):125-44. https://doi.org/http://dx.doi.org/10.4067/S0718-090X2014000100006.

Borges, Fabián A. 2014. "Rules of Procedure as a Cause of Legislative Paralysis: The Case of Costa Rica, 2002-2012". Latin American Politics \& Society 56 (4):119-42. https://doi.org/10.1111/j.15482456.2014.00251.x.

Cascante, Luis Fernando. 2016. "Juan Diego Castro: 'A este país no lo arregla nadie"'. LaRepublica.net - Soluciones para profesionales, el 20 de octubre de 2016. https://www.larepublica.net/noticia/juan-diegocastro-a-este-pas-no-lo-arregla-nadie.

CIEP. 2013. "Estudio de Opinión Sociopolítica, agosto 2013". Centro de Investigación y Estudios Políticos, UCR. http://www.ciep.ucr.ac.cr/index.php/publicaciones/estudiosopinion/Encuesta-de-Opini\%C3\%B3n-Agosto-del-2013/.

CIEP. 2014. "Estudio de Opinión Sociopolítica, enero 2014". Centro de Investigación y Estudios Políticos, UCR. http://www.ciep.ucr.ac.cr/index.php/publicaciones/estudiosopinion/Encuesta-de-Opini\%C3\%B3n-Enero-del-2014/. 
CIEP. 2017. "Estudio de Opinión Sociopolítica, octubre 2017". Centro de Investigación y Estudios Políticos, UCR. http://www.ciep.ucr.ac.cr/index.php/publicaciones/estudiosopinion/Encuesta-de-Opini\%C3\%B3n-Enero-del-2014/.

Díaz González, José Andrés. 2011. "Análisis de la gestión legislativa en Costa Rica (1962-2010): la distribución del poder y la experiencia parlamentaria". Anuario del Centro de Investigaciones y Estudios Políticos 2 (1):70-100.

Díaz González, José Andrés. 2014. "Activos, pasivos y apáticos: imaginarios de la población costarricense sobre la política y la democracia". Revista Rupturas 4 (2):100-123.

Díaz González, José Andrés, y Martín Solís Salazar. 2010. "Percepciones de la ciudadanía costarricense en la primera década del siglo XXI sobre Política y Democracia". En . Heredia: Facultad de Filosofía y Letras, UNA.

Gómez-Campos, Steffan, y Evelyn Villarreal. 2013. "Costa Rica: La Derrota Prematura De Un Gobierno Dividido". Costa Rica: Early Defeat of a Divided Government. 33 (1):117-34.

Hernández Naranjo, Gerardo. 2011. "Partidos Políticos y calidad de la democracia en un contexto de cambios en el sistema de Partidos de Costa RIca". Anuario del Centro de Investigaciones y Estudios Políticos 2:124-47.

Llobet Rodríguez, Javier. 2016. “El 'éxito' del populismo punitivo en Costa Rica y sus consecuencias". Revista Digital de la Maestría en Ciencias Penales. 8 (8). www.revistacienciaspenales.ucr.ac.cr.

Madrigal, Luis Manuel. 2017. "Juan Diego Castro veta a EL MUNDO del anuncio de su candidatura presidencial". EL MUNDO, el 20 de mayo de 2017. https://www.elmundo.cr/juan-diego-castro-veta-a-el-mundodel-anuncio-de-su-candidatura-presidencial/.

Raventós Vorst, Ciska, Marco Vinicio Fournier Facio, Diego Fernández Montero, y Ronald Alfaro Redondo. 2014. Respuestas ciudadanas ante el malestar con la política: Salida, voz y lealtad. San José: EUCR.

Redacción. 2016. "Juan Diego Castro dispara contra periodistas | Crhoy.com". CRHoy.com I, el 21 de octubre de 2016. https://www.crhoy.com/entretenimiento/cultura/juan-diego-castrodispara-contra-periodistas/.

Redacción. 2017a. "Juan Diego Castro: ‘Al diputado que agarre con chorizo, voy con la policía y lo meto a la cárcel'”. AMPrensa.com (blog). el 25 de septiembre de 2017. http://www.amprensa.com/2017/09/juandiego-castro-al-diputado-agarre-chorizo-voy-la-policia-lo-meto-lacarcel/. 
Redacción. 2017b. "Periodista desmiente a candidato presidencial Juan Diego Castro". CRHoy.com, el 24 de noviembre de 2017. https://www.crhoy.com/nacionales/periodista-desmiente-a-candidatopresidencial-juan-diego-castro/.

Mora Solano, Sindy, Martín Solís Salazar y Luis Diego Soto Kiewit. 2014. "Entre El Apoyo a La Democracia Y El Autoritarismo En Costa Rica". Anuario de Estudios Centroamericanos 40:37-60.

Ulloa, César. 2017. El populismo en escena ¿Por qué emerge en unos países y en otros no? $1^{a}$. Quito: FLACSO-Ecuador.

"Un gobierno serio y enérgico". s/f. Juan Diego Presidente 2018 - 2022. Consultado el 28 de noviembre de 2017. https://juandiegopresidente.cr/gobernermos-en-serio/un-gobiernoserio-y-energico/. 